\title{
On the global well-posedness of discrete Boltzmann systems with chemical reaction
}

\author{
Filipe Oliveira*and A. J. Soares ${ }^{\dagger}$ \\ Centro de Matemática, University of Minho \\ Campus of Gualtar, 4710-057 Braga \\ Portugal
}

\begin{abstract}
We study an initial-boundary value problem in one-space dimension for the discrete Boltzmann equation extended to a diatomic gas undergoing both elastic multiple collisions and chemical reactions. By integration of conservation equations, we prove a global existence result in the half-space for small initial data $N_{0} \in \mathcal{B}_{+}^{1} \cap L^{1}$.
\end{abstract}

AMS Classification Numbers: 76P05, 35A05, 35L50

Keywords: Discrete Boltzmann Equation, Chemically reacting gases, Hyperbolic PDEs

\section{Introduction}

In recent years, increasing attention has been devoted to Boltzmann type models for gases with inelastic molecular interactions, due to the presence of chemically reacting mixtures in several applications as combustion engines, chemical reactors and plasma physics. Let us quote, for example, papers [6], [7], [16] in the context of the full Boltzmann Equation (BE), and papers [8], [11], [13], [15] in the context of the Discrete Boltzmann Equation (DBE). This discrete model consists of a system of hyperbolic semi-linear partial differential equations describing the time-space evolution of a gas whose particles can attain a finite number of preselected velocities only ([5]). For what concerns the initialboundary value problem within the DBE, a rather vast bibliography is available in the context of inert gases (see for instance [4], [9], [10]). However, only few results are available in the literature concerning reacting gases. In this context, in [17], an existence result is proven for a particular model concerning a chemically active gas $([11])$.

As pointed out in [1], in spite of the simple mathematical structure of the DBE, the developments of the last decades have shown that the results on existence and uniqueness

*filipeo@math.uminho.pt

†ajsoares@math.uminho.pt 
of solutions are, generally, weaker than the corresponding ones for the BE. Nevertheless, when dealing with fluid dynamical applications and chemistry and physics of moderately dense gases, models of DBE provide an accurate description of the flow pattern, quite suitable for numerical implementations. This justifies the renewed interest in models of DBE extended to chemically reacting gases, several years after the pioneering papers of Broadwell [2] and Cabannes [3] that constituted a breakthrough in the study of the propagation of shock waves within one component inert gases.

In fact, due to the simple structure of the discrete collision operator and the increasing computer capacities, several numerical simulations have been implemented with the aim of studying rather complex processes. In particular, in [14], the steady detonation problem has been solved by means of the kinetic model of [13] for gases undergoing autocatalytic reversible reactions. In this model, the particles can experience binary and triple elastic collisions and chemical reactions. The associated initial-value problem has been sucessfully studied from a numerical point of view in [12], although no existence theorem is available.

In the present paper, we prove the global wellposedness of the initial boundary value problem in one space dimension for the discrete model of [13].

The inclusion of multiple collisions with chemical reactions represents an additional difficulty, since cubic terms appear in the collision operator and the conservation laws differ from the ones of classical inert gases.

We continue this introduction by presenting the model.

\section{Kinetic equations}

$$
\begin{cases}\frac{\partial}{\partial t} N_{i}^{A}(x, t)+v_{i} \frac{\partial}{\partial x} N_{i}^{A}(x, t)=F_{i}^{A}(N(x, t)) & (i \in\{1 ; 2 ; 3\}), \\ \frac{\partial}{\partial t} N_{i}^{A_{2}}(x, t)+\frac{v_{i}}{2} \frac{\partial}{\partial x} N_{i}^{A_{2}}(x, t)=F_{i}^{A_{2}}(N(x, t)) & (i \in\{1 ; 2 ; 3\}), \\ \frac{\partial}{\partial t} N_{i}^{A^{*}}(x, t)+v_{i} \frac{\partial}{\partial x} N_{i}^{A^{*}}(x, t)=F_{i}^{A^{*}}(N(x, t)) & (i \in\{1 ; 3\}),\end{cases}
$$

where $(x, t) \in[0 ;+\infty[\times[0 ;+\infty[$, and the other terms have the following meaning:

- $N=\left(N_{1}^{A}, N_{2}^{A}, N_{3}^{A}, N_{1}^{A^{*}}, N_{3}^{A^{*}}, N_{1}^{A_{2}}, N_{2}^{A_{2}}, N_{3}^{A_{2}}\right)$ is the vector number density associated to particles of a diatomic gas constituted by atoms $A$, accelerated atoms $A^{*}$ and molecules $A_{2}$, subjected to autocatalytic chemical reaction of type

$$
A_{2}+M \rightleftharpoons A+A^{*}+M, \quad M=A, A^{*}, A_{2}
$$

$M$ being the catalyst of the reaction;

- $\left(v_{1}, v_{2}, v_{3}\right)=(c, 0,-c)$, where $c$ is a positive constant defined in terms of the atom mass $m$ and molecular bond energy $\epsilon$ of the diatomic molecule $A_{2}$, through the relation $c=\sqrt{(4 \epsilon) /(5 m)}$; for sake of simplicity, we will assume $m=1$; 
- the collision terms $F_{i}^{M}$ are defined by polynomials of the form

$$
F_{i}^{M}(N)=\left(P_{i, M}^{(1)}(N)+P_{i, M}^{(2)}(N)\right)-N_{i}^{M}\left(Q_{i, M}^{(1)}(N)+Q_{i, M}^{(2)}(N)\right)
$$

which describe the elastic scattering and chemical mechanism among particles; the polynomials $P_{i, M}^{(k)}, Q_{i, M}^{(k)}$ are homogeneous with positive coefficients, $P_{i, M}^{(1)}$ and $Q_{i, M}^{(1)}$ of degree $2, P_{i, M}^{(2)}$ and $Q_{i, M}^{(2)}$ of degree 3 and 1 , respectively. In particular, $P_{i, M}^{(1)}$ and $P_{i, M}^{(2)}$ represent the rate of creation of particles of species $M$ with velocity along $i$-direction due to inert or reactive collisions of binary and triple type, respectively. On the other hand, the terms $N_{i}^{M} Q_{i, M}^{(1)}$ and $N_{i}^{M} Q_{i, M}^{(2)}$ represent the rate of disappearance of particles of species $M$ with velocity along $i$-direction due to inert or reactive collisions of triple and binary type, respectively.

Let us observe that inert collisions involve the same number of incoming and outgoing particles, i.e. two or three particles in correspondence of binary or triple collisions, respectively. Conversely, collisions with chemical reaction (2) lead to the formation of two atoms $A, A^{*}$ and one $M$-particle when a molecule $A_{2}$ dissociates in the presence of the catalist $M$. Therefore, a triple reactive source term corresponds to a binary reactive sink term, and vice-versa. The detailed evaluation of the terms $F_{i}^{M}(N)$ is provided in paper [13].

The particular form of $F_{i}^{M}(N)$ considered in the present paper is appropriate for all those elementary reactions of catalytic type ocurring, for example, in the brain chain of the Hydrogen-Oxygen and Methane combustion mechanisms.

The other case of elementary bimolecular reaction, including only binary encounters and then only quadratic collision terms, has been considered in paper [17], but the existence result is much weaker than the one of the present paper.

Global chemical reactions involving a greater number of gas particles can be considered but, in general, they decompose in a brain chain of elementary reactions of bimolecular and autocatalytic type. In this case, the collision terms obviously become rather cumbersome, but still polynomials, in the unknown number densities, whose degree is equal to the maximum number of the colliding particles. The technique exploited here can also be applied, provided that the reactions are two-way.

\section{Conservation equations}

We assume that (1) possesses the following independent conservation laws (see [13] for details), corresponding, respectively, to the conservation of partial number densities of species $A-A_{2}, A^{*}-A_{2}$ and the total momentum of the system in the $x$-direction.

$$
\frac{\partial}{\partial t}\left(\sum_{i=1}^{3}\left(N_{i}^{A}+N_{i}^{A_{2}}\right)\right)+\frac{\partial}{\partial x}\left(\sum_{i=1}^{3} v_{i}\left(N_{i}^{A}+\frac{1}{2} N_{i}^{A_{2}}\right)\right)=0
$$




$$
\begin{aligned}
\frac{\partial}{\partial t}\left(\sum_{i=1}^{3} N_{i}^{A_{2}}+\sum_{i=1,3} N_{i}^{A^{*}}\right)+\frac{\partial}{\partial x}\left(\sum_{i=1}^{3} \frac{1}{2} v_{i} N_{i}^{A_{2}}+\sum_{i=1,3} v_{i} N_{i}^{A^{*}}\right)=0 \\
\frac{\partial}{\partial t}\left(\sum_{i=1}^{3} v_{i}\left(N_{i}^{A}+N_{i}^{A_{2}}\right)+\sum_{i=1,3} v_{i} N_{i}^{A^{*}}\right) \\
+\frac{\partial}{\partial x}\left(\sum_{i=1}^{3} v_{i}^{2}\left(N_{i}^{A}+\frac{1}{2} N_{i}^{A_{2}}\right)+\sum_{i=1,3} v_{i}^{2} N_{i}^{A^{*}}\right)=0 .
\end{aligned}
$$

\section{Initial-boundary value problem}

We consider the initial-boundary value problem associated to Eqs. (1) with initial data

$$
N_{i}^{M}(x, 0)=N_{i_{0}}^{M}(x) .
$$

where $N_{i_{0}}$ are prescribed functions of $x \geq 0$.

We complete the I.B.V.P. (1)-(6) with the following boundary conditions:

$$
\left(\begin{array}{c}
N_{1}^{A}(0, t) \\
N_{1}^{A^{*}}(0, t) \\
N_{1}^{A_{2}}(0, t)
\end{array}\right)=\left(\begin{array}{ccc}
\beta_{A}^{A} & \beta_{A^{*}}^{A} & \beta_{A_{2}}^{A} \\
\beta_{A}^{A^{*}} & \beta_{A^{*}}^{A^{*}} & \beta_{A_{2}}^{A^{*}} \\
\beta_{A}^{A_{2}} & \beta_{A^{*}}^{A_{2}} & \beta_{A_{2}}^{A_{2}}
\end{array}\right)\left(\begin{array}{c}
N_{3}^{A}(0, t) \\
N_{3}^{A^{*}}(0, t) \\
N_{3}^{A_{2}}(0, t)
\end{array}\right), \quad \forall t>0
$$

with

$$
\sum_{M^{\prime}} \beta_{M^{\prime}}^{M} \leq 1, \quad \beta_{M}^{A}+\beta_{M}^{A^{*}}+\frac{1}{2} \beta_{M}^{A_{2}} \leq \delta_{M},
$$

where $\delta_{M}=1$ if $M=A, A^{*}$ and $\delta_{A_{2}}=\frac{1}{2}$.

Conditions (7) mean that the contribution to the incoming flux of each fixed species $A, A^{*}, A_{2}$ on the boundary at $x=0$ is given by a fraction of outgoing molecules of the same species that have been reflected back from the boundary, and a fraction of molecules of other species that have been reacted.

Moreover, condition (8) assures that the macroscopic flow of the gas is not inward to the boundary, since

$$
\begin{aligned}
\text { Netflux } & =\sum_{i=1,3} v_{i}\left[N_{i}^{A}(0, t)+N_{i}^{A^{*}}(0, t)+\frac{1}{2} N_{i}^{A_{2}}(0, t)\right] \\
& =\sum_{M}\left(\beta_{M}^{A}+\beta_{M}^{A^{*}}+\frac{1}{2} \beta_{M}^{A_{2}}-\delta_{M}\right) N_{3}^{M}(0, t) \\
& \leq 0
\end{aligned}
$$




\section{Compatibility conditions}

Finally, we suppose that the initial data satisfies the following compatibility conditions:

$$
\left(\begin{array}{c}
N_{1_{0}}^{A}(0) \\
N_{1_{0}^{*}}^{A^{*}}(0) \\
N_{1_{0}}^{A_{2}}(0)
\end{array}\right)=\left(\begin{array}{ccc}
\beta_{A}^{A} & \beta_{A^{*}}^{A} & \beta_{A_{2}}^{A} \\
\beta_{A}^{A^{*}} & \beta_{A^{*}}^{A^{*}} & \beta_{A_{2}}^{A^{*}} \\
\beta_{A}^{A_{2}} & \beta_{A^{*}}^{A_{2}} & \beta_{A_{2}}^{A_{2}}
\end{array}\right)\left(\begin{array}{c}
N_{3_{0}}^{A}(0) \\
N_{30}^{A_{0}^{*}}(0) \\
N_{3_{0}}^{A_{2}}(0)
\end{array}\right)
$$

and

$$
\left(\begin{array}{c}
\frac{d}{d x} N_{1_{0}}^{A}(0) \\
\frac{d}{d x} N_{1_{0}}^{A^{*}}(0) \\
\frac{d}{d x} N_{1_{0}}^{A_{2}}(0)
\end{array}\right)=\left(\begin{array}{ccc}
\beta_{A}^{A} & \beta_{A^{*}}^{A} & \beta_{A_{2}}^{A} \\
\beta_{A}^{A^{*}} & \beta_{A^{*}}^{A^{*}} & \beta_{A_{2}}^{A^{*}} \\
\beta_{A}^{A_{2}} & \beta_{A^{*}}^{A_{2}} & \beta_{A_{2}}^{A_{2}}
\end{array}\right)\left(\begin{array}{c}
\frac{d}{d x} N_{3_{0}}^{A}(0) \\
\frac{d}{d x} N_{3_{0}}^{A^{*}}(0) \\
\frac{d}{d x} N_{3_{0}}^{A}(0)
\end{array}\right) .
$$

We end this introduction with a few notations:

For $N(x, t)=\left(N_{1}^{A}(x, t), \ldots, N_{3}^{A_{2}}(x, t)\right)$ and $X \subset \mathbb{R}^{+} \times \mathbb{R}^{+}$, we put

$$
L^{\infty}(X)=\left\{N /\|N\|_{X, \infty}=\max _{i, M} \operatorname{esssup}_{(\mathrm{x}, \mathrm{t}) \in \mathrm{X}}\left|\mathrm{N}_{\mathrm{i}}^{\mathrm{M}}(\mathrm{x}, \mathrm{t})\right|<+\infty\right\}
$$

Also, we define the space of bounded continuously differentiable functions with bounded derivatives:

$$
\mathcal{B}^{1}(X)=\left\{f \in C^{1}(X) / f, \frac{\partial f}{\partial x}, \frac{\partial f}{\partial t} \in L^{\infty}(X)\right\}
$$

and we denote by $\mathcal{B}_{+}^{1}(X)$ its positive cone, composed of all positive functions of $\mathcal{B}^{1}(X)$.

As usual, for $N_{0}(x)=\left(N_{1_{0}}^{A}(x), \ldots, N_{3_{0}}^{A_{2}}(x)\right)$, we introduce the space

$$
L^{1}\left(\mathbb{R}^{+}\right)=\left\{N_{0} /\left\|N_{0}\right\|_{1}=\sum_{i, M} \int_{0}^{+\infty}\left|N_{i_{0}}^{M}(x)\right| d \mu(x)<+\infty\right\} .
$$

Finally, for $T>0$ and $N \in L^{\infty}([0 ;+\infty[\times[0 ; T])$, we introduce the upper bound

$$
E(T)=\max _{i, M} \sup _{(x, t) \in \mathbb{R}^{+} \times[0 ; T]}\left|N_{i}^{M}(x, t)\right|, \quad E_{0}=E(0),
$$

and for $N_{0} \in L^{1}\left(\mathbb{R}^{+}\right)$, we put

$$
m_{0}=\int_{0}^{+\infty}\left(\sum_{i=1}^{3}\left(N_{i_{0}}^{A}+2 N_{i_{0}}^{A_{2}}\right)+\sum_{i=1,3} N_{i_{0}}^{A^{*}}\right)(x) d \mu(x)
$$

corresponding to the initial mass of the system. 


\section{Local solutions}

\section{Theorem 2.1}

Let $N_{o} \in \mathcal{B}_{+}^{1}([0 ;+\infty[)$ satisfying the compatibility conditions (9),(10). Then there exists a unique solution

$$
N \in \mathcal{B}_{+}^{1}\left(\left[0 ;+\infty\left[\times\left[0 ; T_{o}\right]\right)\right.\right.
$$

for the mixed-problem (1),(6),(7), where the life-span $T_{o}>0$ depends exclusively on

$$
E_{o}=\max _{i, M} \sup _{x \in \mathbb{R}^{+}}\left|N_{i_{o}}^{M}(x)\right| .
$$

\section{Proof of Theorem 2.1:}

The proof of this theorem follows the lines of Theorem 3.1 in [9], where the existence of local solutions for a dicrete Boltzmann model with binary collisions is treated.

For self-completeness, we sketch here the above mentioned proof:

For convenience, we put

$$
v_{i}^{M}=v_{i} \text { if } M=A, A^{*} \text { and } v_{i}^{A_{2}}=\frac{v_{i}}{2} .
$$

For some parameter $\lambda>0$, we put (1) in the form

$$
\frac{\partial}{\partial t} N_{i}^{M}(x, t)+v_{i}^{M} \frac{\partial}{\partial x} N_{i}^{M}(x, t)+\lambda N_{i}^{M}(x, t)=F_{i}^{M, \lambda}(N(x, t)),
$$

where

$$
F_{i}^{M, \lambda}(N(x, t))=F_{i}^{M}(N(x, t))+\lambda N_{i}^{M}(x, t)
$$

Let $(x, t) \in[0 ;+\infty[\times[0 ;+\infty[$.

If $x-v_{i}^{M} t \geq 0$, integrating (11) along the $v_{i}^{M}$-characteristic through $(x, t)$ leads to

$$
N_{i}^{M}(x, t)=e^{-\lambda t} N_{i_{o}}\left(x-v_{i}^{M} t\right)+\int_{0}^{t} e^{-\lambda(t-\tau)} F_{i}^{M, \lambda}(N)\left(x-v_{i}^{M}(t-\tau), \tau\right) d \tau .
$$

If $x-v_{i}^{M} t<0$, one gets

$$
N_{i}^{M}(x, t)=e^{-\lambda\left(t-t_{i}^{M}\right)} N_{i}^{M}\left(0, t_{i}^{M}\right)+\int_{t_{i}^{M}}^{t} e^{-\lambda(t-\tau)} F_{i}^{M, \lambda}(N)\left(x-v_{i}^{M}(t-\tau), \tau\right) d \tau,
$$

where $t_{i}^{M}=t-\frac{x}{v_{i}^{M}}$ is the intersection of the $v_{i}^{M}$-characteristic with the boundary $x=0$.

Noticing that in this case $v_{i}^{M}>0(i=1)$, condition (7) yields

$$
N_{i}^{M}(x, t)=e^{-\lambda\left(t-t_{i}^{M}\right)} \sum_{M^{\prime}} \beta_{M^{\prime}}^{M} N_{3}^{M^{\prime}}\left(0, t_{i}^{M}\right)+\int_{t_{i}^{M}}^{t} e^{-\lambda(t-\tau)} F_{i}^{M, \lambda}(N)\left(x-v_{i}^{M}(t-\tau), \tau\right) d \tau .
$$


Finally, integrating along the $v_{3}^{M^{\prime}}$-characteristic through $\left(0, t_{i}^{M}\right)$,

$$
\begin{aligned}
N_{i}^{M}(x, t)= & e^{-\lambda t} \sum_{M^{\prime}} \beta_{M^{\prime}}^{M} e^{-\lambda t_{i}^{M}} N_{3_{0}}^{M^{\prime}}\left(-v_{3}^{M^{\prime}} t_{i}^{M}\right) \\
& +e^{-\lambda t} \sum_{M^{\prime}} \beta_{M^{\prime}}^{M} \int_{0}^{t_{i}^{M}} e^{-\lambda\left(t_{i}^{M}-\tau\right)} F_{3}^{M^{\prime}, \lambda}(N)\left(x-v_{3}^{M^{\prime}}(t-\tau), \tau\right) d \tau \\
& +\int_{t_{i}^{M}}^{t} e^{-\lambda(t-\tau)} F_{i}^{M, \lambda}(N)\left(x-v_{i}^{M}(t-\tau), \tau\right) d \tau
\end{aligned}
$$

Now, for $T>0$, we put $\Omega_{T}=\left[0 ;+\infty\left[\times[0 ; T], \Omega_{M, i, T}^{+}=\left\{(x, t) \in \Omega_{T} ; x \geq v_{i}^{M} t\right\}\right.\right.$ and $\Omega_{M, i, T}^{-}=\left\{(x, t) \in \Omega_{T} ; x<v_{i}^{M} t\right\}$.

We introduce the Banach space

$$
X^{1}(T)=\left\{N=\left(N_{i}^{M}\right)_{i, M} \in C^{o}\left(\Omega_{T}\right) \cap L^{\infty}\left(\Omega_{T}\right) ; \frac{\partial N_{i}^{M}}{\partial x} \in C^{o}\left(\Omega_{T}\right) \cap L^{\infty}\left(\Omega_{T}\right)\right\}
$$

equipped with the norm

$$
\|N\|_{1, T}=\|N\|_{\infty, \Omega_{T}}+\left\|\frac{\partial N}{\partial x}\right\|_{\infty, \Omega_{T}} \text { where }\|N\|_{\infty, \Omega_{T}}=\max _{i, M} \sup _{(x, t) \in \Omega_{T}}\left|N_{i, M}(x, t)\right| .
$$

For $E, G>0$ we consider the closed convex subset $S(T, E, G)$ of $X^{1}(T)$ formed by all functions $N \in X^{1}(T)$ such that for all $i, M$,

$$
N_{i}^{M}(x, 0)=N_{i_{o}}^{M}(x), x \in[0 ;+\infty[
$$

and

$$
0 \leq N_{i}^{M}(x, t) \leq E,\left|\frac{\partial N_{i}^{M}}{\partial x}(x, t)\right| \leq G,(x, t) \in \Omega_{T} .
$$

Using the compatibility conditions $(9),(10)$ and the fact that all the nonlinear terms involved in (1) are Lipschitz (see [9] for details) one can prove that the map

$$
\psi_{\lambda}: N \rightarrow \psi_{\lambda}(N)
$$

given by

$$
\begin{aligned}
\psi_{\lambda}(N)_{i}^{M}(x, t)= & e^{-\lambda t} N_{i_{o}}\left(x-v_{i}^{M} t\right) \\
& +\int_{0}^{t} e^{-\lambda(t-\tau)} F_{i}^{M, \lambda}(N)\left(x-v_{i}^{M}(t-\tau), \tau\right) d \tau \quad \text { if }(x, t) \in \Omega_{M, i, T}^{+},
\end{aligned}
$$


and

$$
\begin{aligned}
\psi_{\lambda}(N)_{i}^{M}(x, t)= & e^{-\lambda t} \sum_{M^{\prime}} \beta_{M^{\prime}}^{M} e^{-\lambda t_{i}^{M}} N_{3_{o}}^{M^{\prime}}\left(-v_{3}^{M^{\prime}} t_{i}^{M}\right) \\
& +e^{-\lambda t} \sum_{M^{\prime}} \beta_{M^{\prime}}^{M} \int_{0}^{t_{i}^{M}} e^{-\lambda\left(t_{i}-\tau\right)} F_{3}^{M^{\prime}, \lambda}(N)\left(x-v_{3}^{M^{\prime}}(t-\tau), \tau\right) d \tau \\
& \left.+\int_{t_{i}^{M}}^{t} e^{-\lambda(t-\tau)} F_{i}^{M, \lambda}(N)\left(x-v_{i}^{M}(t-\tau)\right), \tau\right) d \tau \quad \text { if }(x, t) \in \Omega_{M, i, T}^{-}
\end{aligned}
$$

is a contraction of $S(T, E, G)$ for adequate values of $\lambda, T, E$, and $G$, where $\lambda, T$ and $E$ depend exclusively on $E_{o}$.

Then, the unique fix-point $N$ of $\psi_{\lambda}$ is a local solution of (1), with

$$
N \in C^{0}\left(\Omega_{T}\right) \cap L^{\infty}\left(\Omega_{T}\right) \quad \text { and } \quad \frac{\partial N}{\partial x} \in C^{0}\left(\Omega_{T}\right) \cap L^{\infty}\left(\Omega_{T}\right) .
$$

It is a technicality to prove that $N$ is continuously differentiable with respect to $t$ and that $N$ solves Eq.(1). The reader can refer to [9] (proof of Theorem 3.1).

\section{Global solutions}

We consider the new set of variables

$$
\begin{aligned}
& M_{1}=N_{1}^{A}+N_{1}^{A^{*}} \\
& M_{2}=N_{1}^{A_{2}} \\
& M_{3}=N_{2}^{A}+N_{2}^{A_{2}} \\
& M_{4}=N_{3}^{A_{2}} \\
& M_{5}=N_{3}^{A}+N_{3}^{A^{*}} .
\end{aligned}
$$

This new set satisfies the system

$$
\frac{\partial}{\partial t} M_{i}(x, t)+w_{i} \frac{\partial}{\partial x} M_{i}(x, t)=R_{i}(N(x, t)), \quad i \in\{1 ; \ldots ; 5\},
$$

where $\left(w_{1}, w_{2}, w_{3}, w_{4}, w_{5}\right)=\left(c, \frac{c}{2}, 0,-\frac{c}{2},-c\right)$ and

$$
\begin{aligned}
& R_{1}=F_{1}^{A}+F_{1}^{A^{*}} \\
& R_{2}=F_{1}^{A_{2}} \\
& R_{3}=F_{2}^{A}+F_{2}^{A_{2}} \\
& R_{4}=F_{3}^{A_{2}} \\
& R_{5}=F_{3}^{A}+F_{3}^{A^{*}} .
\end{aligned}
$$


We make the usual following assumption: for every $j \in\{1, \ldots, 5\}, R_{j}$ can be put in the form

$$
R_{j}(N)=N_{i}^{M} R_{j}^{(1)}-R_{j}^{(2)},
$$

where $v_{i}^{M} \neq w_{j}$ and $R^{(k)}, k=1,2$ are polynomials with positive cefficients. Note that this condition holds in the systems studied in [12].

Under this assumption, we prove in this section the following theorem:

\section{Theorem 3.1}

Let $N_{o} \in \mathcal{B}_{+}^{1}\left(\left[0 ;+\infty[) \cap L^{1}([0 ;+\infty[)\right.\right.$ satisfying the compatibility conditions (9)-(10).

Then there exists $\epsilon, \epsilon^{\prime}>0$ such that if

$m_{o}=\int_{0}^{+\infty}\left(\sum_{i=1}^{3}\left(N_{i_{o}}^{A}+2 N_{i_{o}}^{A_{2}}\right)+\sum_{i=1,3} N_{i_{o}}^{A^{*}}\right)(x) d x<\epsilon \quad$ and $E_{o}=\max _{i, M} \sup _{x \in \mathbb{R}^{+}}\left|N_{i_{o}}^{M}(x)\right|<\epsilon^{\prime}$,

the mixed problem (1)-(6)-(7) has a unique solution

$$
N \in \mathcal{B}_{+}^{1}([0 ;+\infty[\times[0 ;+\infty[) .
$$

\section{Proof:}

Let $N_{o} \in \mathcal{B}_{+}^{1}\left(\left[0 ;+\infty[) \cap L^{1}(] 0 ;+\infty[)\right.\right.$ satisfying $(9)$-(10) and $N \in \mathcal{B}_{+}^{1}\left(\left[0 ;+\infty\left[\times\left[0 ; T_{o}\right]\right)\right.\right.$ the solution to (1)-(6)-(7) given by Theorem 2.1 .

In order to establish Theorem 3.1, we prove the following key lemma:

Lemma 3.2 For all $t \in\left[0 ; T_{o}\right]$,

$$
E(t) \leq C E_{o}+C^{\prime} m_{o}\left(E(t)+E(t)^{2}\right)
$$

where the constants $C, C^{\prime}$ are independent of $T_{o}$ and $N_{o}$.

\section{Proof of Lemma 3.2:}

Following the ideas in [4] and [10], the proof of this lemma is based on the integration of the conservation laws $(3),(4),(5)$ along closed curves formed by the characteristic lines of (1).

In what follows we will denote indifferently by $C$ and $C^{\prime}$ some positive constants.

Let $X=(x, t) \in \mathbb{R}^{+} \times \mathbb{R}^{+}$. We begin by considering the case $c t-x>0$. 
In this case the $c$-characteristic through $X$ meets the boundary $x=0$ at a time $t_{c}=t-\frac{x}{c}>0$.

Let $j \in\{1 ; \ldots ; 5\}$.

We first assume that $x-w_{j} t<0$.

In this case $w_{j} \in\left\{\frac{c}{2}, c\right\}$ (i.e. $j \in\{1,2\}$ ) and the characteristic corresponding to the speed $w_{j}$ meets the boundary $x=0$ at the time $t_{j}=t-\frac{x}{w_{j}}$. Integrating (17) along this line,

$$
M_{j}(x, t)=M_{j}\left(0, t_{j}\right)+\int_{t_{j}}^{t} R_{j}\left(N\left(x-w_{j}(t-\tau), \tau\right)\right) d \tau .
$$

Also, by (7),

$$
M_{j}\left(0, t_{j}\right) \leq \sum_{M, M^{\prime}} \beta_{M}^{M^{\prime}} N_{3}^{M}\left(0, t_{j}\right) \leq C\left(M_{4}\left(0, t_{j}\right)+M_{5}\left(0, t_{j}\right)\right)
$$

Hence

$$
\begin{aligned}
M_{j}(x, t) & \leq C\left(M_{4}\left(0, t_{j}\right)+M_{5}\left(0, t_{j}\right)\right)+\int_{t_{j}}^{t} R_{j}\left(N\left(x-w_{j}(t-\tau), \tau\right)\right) d \tau \\
& \leq C\left(M_{4}\left(0, t_{j}\right)+M_{5}\left(0, t_{j}\right)\right)+C^{\prime}\left(E(t)+E(t)^{2}\right) \int_{t_{j}}^{t} \sum_{v_{i}^{M} \neq w_{j}} N_{i}^{M}\left(x-w_{j}(t-\tau), \tau\right) d \tau,
\end{aligned}
$$

where we have used condition (18).

We now estimate $I=\int_{t_{j}}^{t} \sum_{v_{i}^{M} \neq w_{j}} N_{i}^{M}\left(x-w_{j}(t-\tau), \tau\right) d \tau$.

Let us consider the differential form

$$
w=-\left(\sum_{i=1}^{3}\left(N_{i}^{A}+2 N_{i}^{A_{2}}\right)+\sum_{i=1,3} N_{i}^{A^{*}}\right) d x+\left(\sum_{i=1}^{3} v_{i}\left(N_{i}^{A}+N_{i}^{A_{2}}\right)+\sum_{i=1,3} v_{i} N_{i}^{A^{*}}\right) d t .
$$

By making use of the conservation laws (3),(4) we easily check that $w$ is an exact form, hence by putting $A=\left(0, t_{c}\right)$ and $B=(x+c t, 0)$,

$$
\int_{[O A]} w+\int_{[A X]} w+\int_{[X B]} w+\int_{[B O]} w=0 .
$$




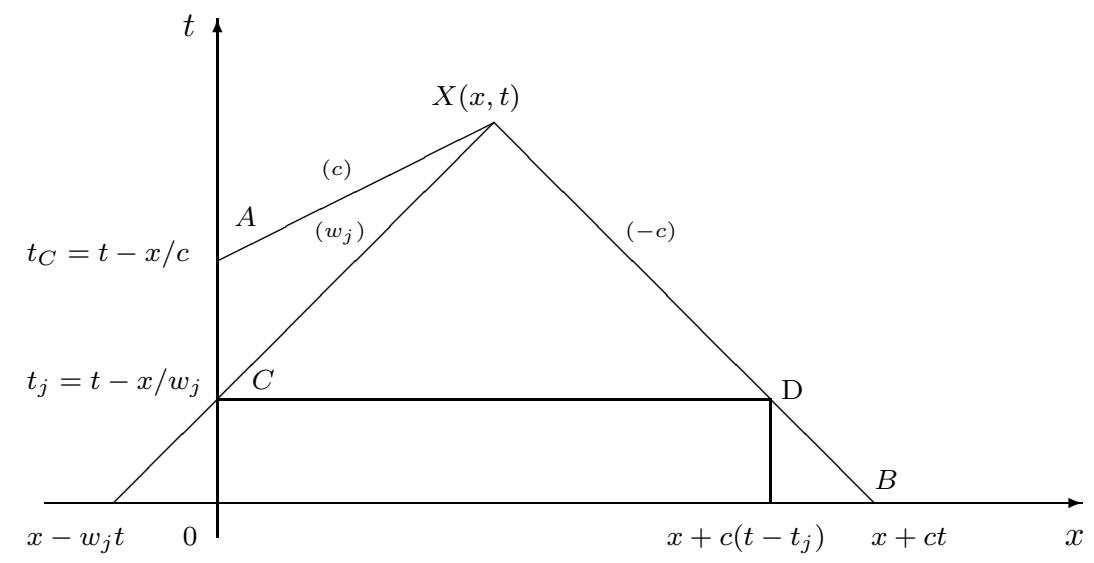

Furthermore,

$$
\int_{[O A]} w=\int_{0}^{t_{c}}\left(\sum_{i=1}^{3} v_{i}\left(N_{i}^{A}+N_{i}^{A_{2}}\right)+\sum_{i=1,3} v_{i} N_{i}^{A^{*}}\right)(0, \tau) d \tau \leq 0,
$$

by the boundary condition (7).

Also,

$$
\begin{gathered}
\int_{[A X]} w=\int_{t_{c}}^{t}\left(\sum_{i=1}^{3}\left(\left(v_{i}-c\right) N_{i}^{A}+\left(v_{i}-2 c\right) N_{i}^{A_{2}}\right)+\sum_{i=1,3}\left(v_{i}-c\right) N_{i}^{A^{*}}\right)(x-c(t-\tau), \tau) d \tau \\
\int_{[X B]} w=-\int_{0}^{t}\left(\sum_{i=1}^{3}\left(\left(v_{i}+c\right) N_{i}^{A}+\left(v_{i}+2 c\right) N_{i}^{A_{2}}\right)+\sum_{i=1,3}\left(v_{i}+c\right) N_{i}^{A^{*}}\right)(x+c(t-\tau), \tau) d \tau
\end{gathered}
$$

and

$$
\int_{[B O]} w=\int_{0}^{x+c t}\left(\sum_{i=1}^{3}\left(N_{i}^{A}+2 N_{i}^{A_{2}}\right)+\sum_{i=1,3} N_{i}^{A^{*}}\right)(x+c t-\xi, 0) d \xi \leq m_{o} .
$$

By (22),

$$
\begin{aligned}
& \int_{0}^{t}\left(\sum_{i=1}^{3}\left(\left(v_{i}+c\right) N_{i}^{A}+\left(v_{i}+2 c\right) N_{i}^{A_{2}}\right)+\sum_{i=1,3}\left(v_{i}+c\right) N_{i}^{A^{*}}\right)(x+c(t-\tau), \tau) d \tau+ \\
& +\int_{t_{c}}^{t}\left(\sum_{i=1}^{3}\left(\left(c-v_{i}\right) N_{i}^{A}+\left(2 c-v_{i}\right) N_{i}^{A_{2}}\right)+\sum_{i=1,3}\left(c-v_{i}\right) N_{i}^{A^{*}}\right)(x-c(t-\tau), \tau) d \tau \leq m_{o}
\end{aligned}
$$


and

$$
\int_{0}^{t}\left(\sum_{i=1,2} N_{i}^{A}+\sum_{i=1}^{3} N_{i}^{A_{2}}+N_{1}^{A^{*}}\right)(x+c(t-\tau), \tau) d \tau \leq C m_{o} .
$$

We now consider $C=\left(0, t_{j}\right)$ and $D=\left(x+c\left(t-t_{j}\right), t_{j}\right)$.

As before,

$$
\int_{[O C]} w+\int_{[C D]} w+\int_{[D B]} w+\int_{[B O]} w=0 .
$$

An elementary computation leads to

$$
\begin{gathered}
\int_{[O C]} w=\int_{0}^{t_{j}}\left(\sum_{i=1}^{3} v_{i}\left(N_{i}^{A}+N_{i}^{A_{2}}\right)+\sum_{i=1,3} v_{i} N_{i}^{A^{*}}\right)(0, \tau) d \tau \leq 0 \\
\int_{[C D]} w=-\int_{0}^{x+c\left(t-t_{j}\right)}\left(\sum_{i=1}^{3}\left(N_{i}^{A}+2 N_{i}^{A_{2}}\right)+\sum_{i=1,3} N_{i}^{A^{*}}\right)\left(\xi, t_{j}\right) d \xi \\
\int_{[D B]} w=-\int_{0}^{t_{j}}\left(\sum_{i=1}^{3}\left(\left(v_{i}+c\right) N_{i}^{A}+\left(v_{i}+2 c\right) N_{i}^{A_{2}}\right)+\sum_{i=1,3}\left(v_{i}+c\right) N_{i}^{A^{*}}\right)(x+c(t-\tau), \tau) d \tau \\
\leq 0 .
\end{gathered}
$$

By (29),

$$
\int_{0}^{x+c\left(t-t_{j}\right)}\left(\sum_{i=1}^{3}\left(N_{i}^{A}+2 N_{i}^{A_{2}}\right)+\sum_{i=1,3} N_{i}^{A^{*}}\right)\left(\xi, t_{j}\right) d \xi \leq m_{o} .
$$

Next, we consider the differential form $w^{\prime}$ defined by

$$
\begin{aligned}
w^{\prime}= & -\left(\sum_{i=1}^{3}\left(\left(w_{j}-v_{i}\right) N_{i}^{A}+\left(2 w_{j}-v_{i}\right) N_{i}^{A_{2}}\right)+\sum_{i=1,3}\left(w_{j}-v_{i}\right) N_{i}^{A^{*}}\right) d x \\
& +\left(\sum_{i=1}^{3} v_{i}\left(\left(w_{j}-v_{i}\right) N_{i}^{A}+\left(w_{j}-\frac{1}{2} v_{i}\right) N_{i}^{A_{2}}\right)+\sum_{i=1,3} v_{i}\left(w_{j}-v_{i}\right) N_{i}^{A^{*}}\right) d t .
\end{aligned}
$$

By (5), $w^{\prime}$ is an exact form.

$$
\begin{aligned}
& \int_{[X C]} w^{\prime}= \\
& \int_{t_{j}}^{t}\left(\sum_{i=1}^{3}\left(\left(w_{j}-v_{i}\right)^{2} N_{i}^{A}+2\left(w_{j}-\frac{1}{2} v_{i}\right)^{2} N_{i}^{A_{2}}\right)+\sum_{i=1,3}\left(w_{j}-v_{i}\right)^{2} N_{i}^{A^{*}}\right)\left(x-w_{j}(t-\tau), \tau\right) d \tau
\end{aligned}
$$




$$
\int_{[C D]} w^{\prime}=-\int_{0}^{x+c\left(t-t_{j}\right)}\left(\sum_{i=1}^{3}\left(\left(w_{j}-v_{i}\right) N_{i}^{A}+\left(2 w_{j}-v_{i}\right) N_{i}^{A_{2}}\right)+\sum_{i=1,3}\left(w_{j}-v_{i}\right) N_{i}^{A^{*}}\right)\left(\xi, t_{j}\right) d \tau
$$

and

$$
\begin{aligned}
& \int_{[D X]} w^{\prime}= \\
& \int_{t_{j}}^{t} c\left(\sum_{i=1}^{3}\left(\left(w_{j}-v_{i}\right) N_{i}^{A}+\left(2 w_{j}-v_{i}\right) N_{i}^{A_{2}}\right)+\sum_{i=1,3}\left(w_{j}-v_{i}\right) N_{i}^{A^{*}}\right)(x+c(t-\tau), \tau) d \tau+ \\
& +\int_{t_{j}}^{t}\left(\sum_{i=1}^{3} v_{i}\left(\left(w_{j}-v_{i}\right) N_{i}^{A}+\left(w_{j}-2 v_{i}\right) N_{i}^{A_{2}}\right)+\sum_{i=1,3} v_{i}\left(w_{j}-v_{i}\right) N_{i}^{A^{*}}\right)(x+c(t-\tau), \tau) d \tau \\
& =\int_{t_{j}}^{t}\left(\sum_{i=1}^{3}\left(\left(\left(v_{i}+c\right)\left(w_{j}-v_{i}\right) N_{i}^{A}+2\left(w_{j}-\frac{v_{i}}{2}\right)\left(c+\frac{v_{i}}{2}\right) N_{i}^{A_{2}}\right)\right)((x+c(t-\tau), \tau)) d \tau+\right. \\
& +\sum_{i=1,3}\left(\left(v_{i}+c\right)\left(w_{j}-v_{i}\right) N_{i}^{A^{*}}\right)((x+c(t-\tau), \tau)) d \tau . \quad
\end{aligned}
$$

Hence,

$$
\begin{aligned}
\int_{[X C]} w^{\prime}= & -\int_{[C D]} w^{\prime}-\int_{[D X]} w^{\prime} \\
\leq & \left|\int_{[C D]} w^{\prime}\right|+\left|\int_{[D X]} w^{\prime}\right| \\
\leq & C \int_{0}^{x+c\left(t-t_{j}\right)}\left(\sum_{i=1}^{3}\left(N_{i}^{A}+2 N_{i}^{A_{2}}\right)+\sum_{i=1,3} N_{i}^{A^{*}}\right)\left(\xi, t_{j}\right) d \xi \\
& +C \int_{0}^{t}\left(\sum_{i=1,2} N_{i}^{A}+\sum_{i=1}^{3} N_{i}^{A_{2}}+N_{1}^{A^{*}}\right)(x+c(t-\tau), \tau) d \tau \\
\leq & C m_{o}
\end{aligned}
$$

by (28) and (33).

Finally, by (35),

$$
I=\int_{t_{j}}^{t} \sum_{v_{i}^{M} \neq w_{j}} N_{i}^{M}\left(x-w_{j}(t-\tau), \tau\right) d \tau \leq C m_{o}
$$


Next, we consider the case where $x_{j}=x-w_{j} t \geq 0$.

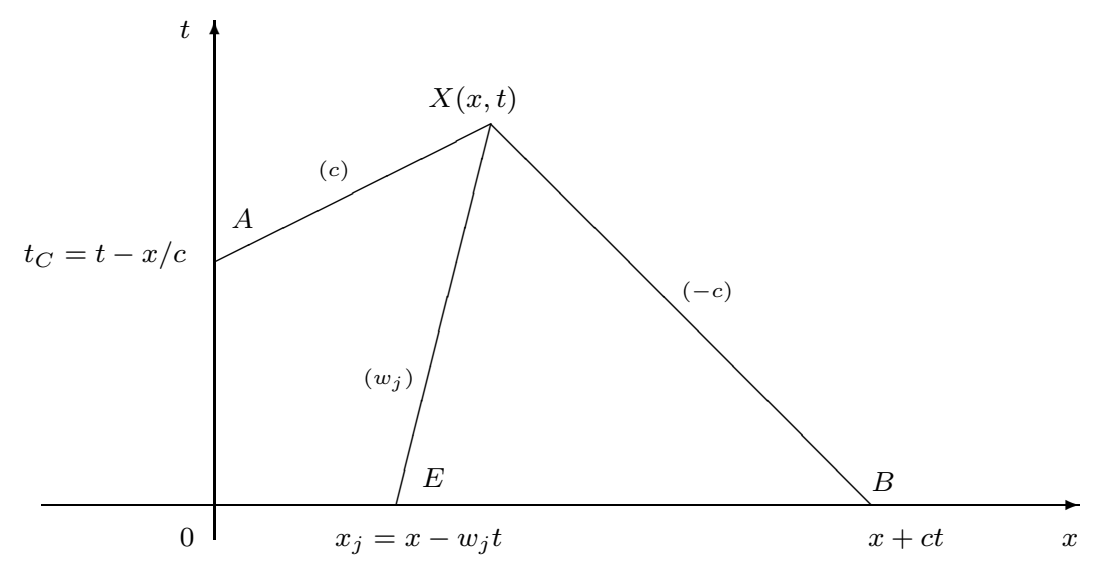

In this case the characteristic corresponding to a speed $w_{j}$ meets $t=0$ at $x_{j}=x-w_{j} t$. We put $E=\left(x_{j}, 0\right)$.

Here, for $j \in\{1 ; \ldots 5\}$,

$$
M_{j}(x, t)=M_{j}\left(x_{j}, 0\right)+\int_{0}^{t} R_{j}\left(N\left(x-w_{j}(t-\tau), \tau\right)\right) d \tau,
$$

and, as before,

$$
M_{j}(x, t) \leq C E_{o}+C^{\prime}\left(E(t)+E(t)^{2}\right) \int_{0}^{t} \sum_{v_{i}^{M} \neq w_{j}} N_{i}^{M}\left(x-w_{j}(t-\tau), \tau\right) d \tau
$$

In order to estimate $J=\int_{0}^{t} \sum_{v_{i}^{M} \neq w_{j}} N_{i}^{M}\left(x-w_{j}(t-\tau), \tau\right) d \tau$, we integrate $w^{\prime}$ on the triangle EXB:

$$
\begin{aligned}
& \int_{[X E]} w^{\prime}= \\
& \int_{0}^{t}\left(\sum_{i=1}^{3}\left(\left(w_{j}-v_{i}\right)^{2} N_{i}^{A}+2\left(w_{j}-\frac{v_{i}^{2}}{2}\right) N_{i}^{A_{2}}\right)+\sum_{i=1,3}\left(w_{j}-v_{i}\right)^{2} N_{i}^{A^{*}}\right)\left(x-w_{j}(t-\tau), \tau\right) d \tau
\end{aligned}
$$


and

$$
\begin{aligned}
& \left|\int_{[E B]} w^{\prime}\right|+\left|\int_{[B X]} w^{\prime}\right| \leq \\
& \quad \int_{x_{j}}^{x}\left(\sum_{i=1}^{3}\left(\left(w_{j}-v_{i}\right) N_{i}^{A}+\left(2 w_{j}-v_{i}\right) N_{i}^{A_{2}}\right)+\sum_{i=1,3}\left(w_{j}-v_{i}\right) N_{i}^{A^{*}}\right)(\xi, 0) d \tau+ \\
& +\int_{0}^{t}\left(\sum_{i=1}^{3}\left(\left(\left(v_{i}+c\right)\left(w_{j}-v_{i}\right) N_{i}^{A}+2\left(w_{j}-\frac{v_{i}}{2}\right)\left(c+\frac{v_{i}}{2}\right) N_{i}^{A_{2}}\right)\right)\right)((x+c(t-\tau), \tau)) d \tau+ \\
& +\sum_{i=1,3}\left(\left(v_{i}+c\right)\left(w_{j}-v_{i}\right) N_{i}^{A^{*}}\right)((x+c(t-\tau), \tau)) d \tau,
\end{aligned}
$$

hence

$$
\begin{aligned}
&\left|\int_{[E B]} w^{\prime}\right|+\left|\int_{[B X]} w^{\prime}\right| \leq C m_{o}+ \\
&+C^{\prime} \int_{0}^{t}\left(\sum_{i=1,2} N_{i}^{A}+\sum_{i=1}^{3} N_{i}^{A_{2}}+N_{1}^{A^{*}}\right)(x+c(t-\tau), \tau) d \tau \leq C m_{o}
\end{aligned}
$$

by $(28)$.

Finally,

$$
J=\int_{0}^{t} \sum_{v_{i}^{M} \neq w_{j}} N_{i}^{M}\left(x-w_{j}(t-\tau), \tau\right) d \tau \leq C \int_{[X E]} w^{\prime} \leq C m_{o}
$$

By combining this estimate with (40), we obtain, in the case where $x-w_{j} t \geq 0$,

$$
\forall j, M_{j}(x, t) \leq C E_{o}+C m_{o}\left(E(t)+E(t)^{2}\right) .
$$

If $x-w_{j} t<0$, by $(21)$ and (38),

$$
M_{j}(x, t) \leq C\left(M_{4}\left(0, t_{j}\right)+M_{5}\left(0, t_{j}\right)\right)+C m_{o}\left(E(t)+E(t)^{2}\right) .
$$

For $j \in\{4,5\}$, integration along the characteristic of speed $w_{j}$ leads to

$$
\begin{aligned}
M_{j}\left(0, t_{j}\right) & =M_{j}\left(w_{j} t_{j}, 0\right)+\int_{0}^{t_{j}} R_{j}\left(N\left(-w_{j}\left(\tau-t_{j}\right), \tau\right)\right) d \tau \\
& \leq C E_{o}+\left(E(t)+E(t)^{2}\right) \int_{0}^{t_{j}} \sum_{v_{i}^{M} \neq w_{j}} N_{i}^{M}\left(-w_{j}(t-\tau), \tau\right) d \tau \\
& \leq C E_{o}+C^{\prime} m_{o}\left(E(t)+E(t)^{2}\right)
\end{aligned}
$$


by $(42)$.

Replacing in (44) leads to

$$
M_{j}(x, t) \leq C E_{o}+C m_{o}\left(E(t)+E(t)^{2}\right)
$$

also in the case $x-w_{j} t<0$.

Finally, if the characteristic corresponding to a speed $c$ does not meet the boundary $x=0$, we put $x_{c}=x-c t$ and $F=\left(x_{c}, 0\right)$.

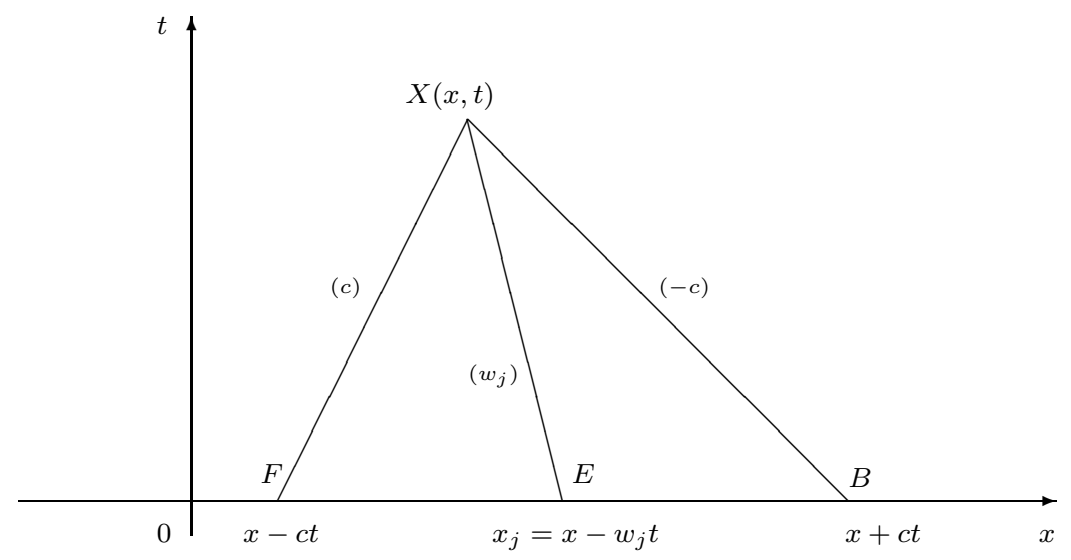

Integrating $w$ on the triangle $X B F$ and $w^{\prime}$ on the triangle $X F E$ leads by analogous computations to (43) which concludes the proof of Lemma 3.2.

We now prove the following lemma:

Lemma 3.3 There exists $\epsilon>0, \epsilon^{\prime}>0$ such that if $m_{o}<\epsilon$ and $E_{o}<\epsilon^{\prime}$ then there exists a constant $K=K\left(N_{o}\right)>0$ depending exclusively on the initial data $N_{o}$ such that

$$
\forall 0 \leq t \leq T_{o}, E(t) \leq K\left(N_{o}\right)
$$

One can easily show by standard arguments that Theorem 3.1 can be obtained from Lemma 3.3.

\section{Proof of Lemma 3.3}

We define the polynomial

$$
P: Y \in \mathbb{R} \rightarrow C^{\prime} m_{o} Y^{2}+\left(C^{\prime} m_{o}-1\right) Y+C E_{o} .
$$


Let $m_{o}<\frac{1}{C^{\prime}}:=\epsilon$ and $\epsilon^{\prime}>0$ small enough such that if $E_{o}<\epsilon^{\prime}$,

$$
\left(C^{\prime} m_{o}-1\right)^{2}-4 C C^{\prime} m_{o} E_{o}>0 .
$$

Then $P$ possesses the two roots

$$
\begin{aligned}
& Y_{1}\left(E_{o}, m_{o}\right)=\frac{1-C^{\prime} m_{o}-\sqrt{\left(C^{\prime} m_{o}-1\right)^{2}-4 C C^{\prime} m_{o} E_{o}}}{C^{\prime} m_{o}}>0, \\
& Y_{2}\left(E_{o}, m_{o}\right)=\frac{1-C^{\prime} m_{o}+\sqrt{\left(C^{\prime} m_{o}-1\right)^{2}-4 C C^{\prime} m_{o} E_{o}}}{C^{\prime} m_{o}}>0 .
\end{aligned}
$$

Since for all $t \in\left[0 ; T_{o}\right], P(E(t)) \geq 0$ and $t \rightarrow E(t)$ is continuous,

$$
\forall 0 \leq t \leq T_{o}, E(t) \leq Y_{1} \text { or } \forall 0 \leq t \leq T_{o}, E(t) \geq Y_{2} .
$$

Clearly, $Y_{2} \leq E_{o}$ cannot hold for $E_{o}$ small enough, since

$$
\lim _{E_{o} \rightarrow 0} Y_{2}\left(E_{o}, m_{o}\right)=2 \frac{1-C^{\prime} m_{o}}{C^{\prime} m_{o}}>0 .
$$

Hence Lemma 3.3 holds with $K\left(N_{o}\right)=Y_{1}\left(E_{o}, m_{o}\right)$.

\section{References}

[1] N. Bellomo and T. Gustafsson, "The discrete Boltzmann equation: a review of the mathematical aspects of the initial-boundary value problems", Review Math. Phys., Vol. 3, No. 2, pp. 137-162, 1991.

[2] J. E. Broadwell, "Shock Structure in a Simple Discrete Velocity Gas", Phys. Fluids, Vol. 7, No. 8, pp. 1243-1247, 1964.

[3] H. Cabannes, "Etude de la Propagation des Ondes dans un Gaz à Quatorze Vitesses", J. Méc., Vol. 4, No. 4, pp. 705-744, 1975.

[4] H. Cabannes, "Global solution of the discrete Boltzmann equation", Eur. J. Mech. B-Fluids, Vol. 11, pp. 415-437, 1992.

[5] R. Gatignol, Théorie cinétique des gaz à répartition discrète des vitesses, Lect. Notes in Phys., 36, Berlin (Springer-Verlag), 1975.

[6] V. Giovangigli, Multicomponent Flow Modeling, Boston, USA: Birkhäuser, 1999.

[7] M. Groppi and G. Spiga, "Kinetic approach to chemical reactions and inelastic transitions in a rarefied gas", J. Math. Chem., Vol. 26, pp. 197-219, 1999.

[8] F. Hanser, W. Koller and F. Schürrer, "Treatment of laser induced thermal acoustics in the framework of discrete kinetic theory", Phys. Rev. E, Vol. 61, pp. 2065$2073,2000$. 
[9] S. Kawashima, "Global solutions to the initial-boundary value problems for the discrete Boltzmann equation", Nonlinear analysis TMA, Vol. 17, pp. 577-597, 1991.

[10] S. Kawasima and H. Cabannes, "Initial value problem in Discrete Kinetic Theory", Pogresses in Astronautics and Aeronautics, Vol. 118, pp. 148-154, 1989.

[11] R. Monaco, M. Pandolfi Bianchi and A. Rossani, "Chapman-Enskog expansion of a discrete velocity model with bi-molecular reactions", Math. Models Meth. Appl. Sci., Vol. 4, pp. 355-370, 1994.

[12] R. Monaco, M. Pandolfi Bianchi, A. J. Soares, "Numerical Simulations of a Boltzmann Model for Reacting Gases", Appl. Math. and Comput., Vol. 85, pp. 61-85, 1997.

[13] M. Pandolfi Bianchi, A. J. Soares, "The discrete Boltzmann equation for gases with autocatalytic reversible reactions", Commun. Appl. Nonlinear. Anal., Vol. 1, pp. 25-48 1994.

[14] M. Pandolfi Bianchi, A. J. Soares, "A kinetic model for a reacting gas flow: steady detonation and speeds of sound", Phys. Fluids, Vol. 8, pp. 3423-3432, 1996.

[15] M. Pandolfi Bianchi and A. J. Soares, "Reactive Euler equations of discrete models with reversible reactions", Continuum Mech. Thermodyn., Vol. 12, pp. 53-67, 2000.

[16] A. Rossani and G. Spiga, "A note on the kinetic theory of chemically reacting gases", Physica A, Vol. 272, pp. 563-573, 1999.

[17] A. J. Soares, "Initial-boundary value problem for the Broadwell model of a gas mixture with bimolecular reaction", Math. Meth. Appl. Sci., Vol. 21, pp. 501-517, 1998. 